\title{
Erratum: Primordial Black Hole Scenario for the Gravitational-Wave Event GW150914 [Phys. Rev. Lett. 117, 061101 (2016)]
}

Misao Sasaki, Teruaki Suyama, Takahiro Tanaka, and Shuichiro Yokoyama

Q (Received 11 July 2018; published 31 July 2018)

DOI: 10.1103/PhysRevLett.121.059901

There was a mistake in computing the merger rate for $\alpha=0.4, \beta=0.8$. Consequently, the dotted (blue) curve in Fig. 1 in the original Letter is incorrect. The corrected version of Fig. 1 is shown below. The main conclusion of the Letter remains unchanged. We thank Dr. Ryan Magee for bringing this to our attention.

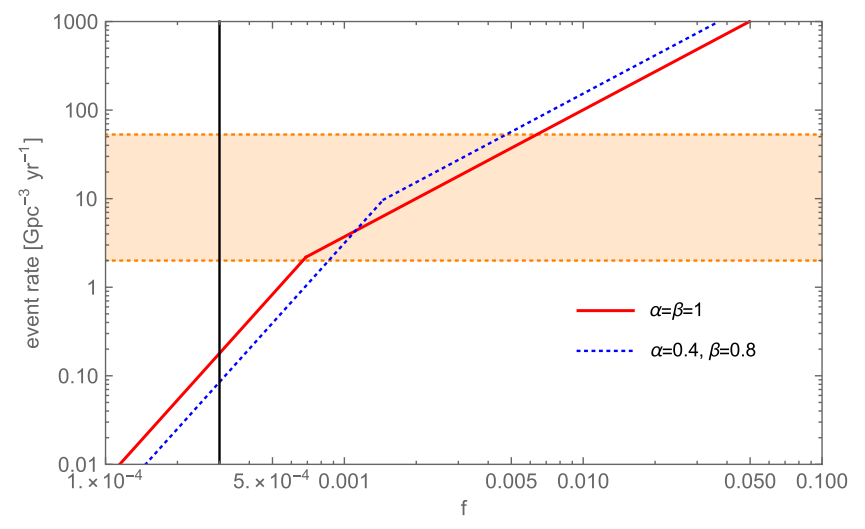

FIG. 1. The dotted (blue) curve is corrected. 